\title{
In vivo photoacoustic assessment of the oxygen saturation changes in the human radial artery: a preliminary study associated with age
}

\author{
Taehoon Bok $\odot^{\text {a,b,c }}{ }^{\text {Eno Hysi, }},{ }^{\mathrm{c}}$ and Michael C. Kolios $\odot^{\mathrm{a}, \mathrm{b}, \mathrm{c}, *}$ \\ ${ }^{a}$ Ryerson University, Faculty of Science, Department of Physics, Toronto, Canada \\ ${ }^{b}$ Institute for Biomedical Engineering, Science and Technology, Toronto, Canada \\ ${ }^{\mathrm{c}}$ St. Michael's Hospital, Keenan Research Centre for Biomedical Science, \\ Division of Nephrology, Toronto, Canada
}

\begin{abstract}
Significance: We demonstrate the potential of probing the $\mathrm{sO}_{2}$ change under blood flow in vivo using photoacoustic (PA) imaging and sheds light on the complex relationship between RBC aggregation and oxygen delivery.

Aim: To conduct in vivo assessments of the $\mathrm{sO}_{2}$ in the radial artery of healthy volunteers and simultaneously probe the relation between the $\mathrm{sO}_{2}$ and hemodynamic behavior such as red blood cell (RBC) aggregation.

Approach: The effects of PA-based measurements of blood hemodynamics were studied as a function of the subjects' age (20s, 30s, and 40s). The pulsatile blood flow in the human radial artery of 12 healthy subjects was imaged in the 700 to $900 \mathrm{~nm}$ optical wavelength range using a linear array-based PA system.

Results: The PA power when blood velocity is minimum $\left(P a_{\max }\right)$ was larger than the one attained at maximum blood velocity $\left(P a_{\min }\right)$, consistent with predictions based on the cyclical variation of RBC aggregation during pulsatile flow. The difference between $P a_{\min }$ and $P a_{\max }$ at $800 \mathrm{~nm}\left(\Delta P a_{800}\right)$ increased with age $(1.7,2.2$, and $2.6 \mathrm{~dB}$ for age group of $20 \mathrm{~s}, 30 \mathrm{~s}$, and $40 \mathrm{~s}$, respectively). The $\mathrm{sO}_{2}$ computed from $P a_{\max }$ was larger than the one from $P a_{\min }$.

Conclusions: The $\triangle P a_{800}$ increased with participant age. The $\Delta P a_{800}$ metric could be a surrogate of noninvasively monitoring the age-induced changes in $\mathrm{RBC}$ aggregation. The $\mathrm{sO}_{2}$ change during a cycle of pulsatile blood flow also increased with age, demonstrating that RBC aggregation can affect the $\mathrm{sO}_{2}$ change.

(C) The Authors. Published by SPIE under a Creative Commons Attribution 4.0 Unported License. Distribution or reproduction of this work in whole or in part requires full attribution of the original publication, including its DOI. [DOI: 10.1117/1.JBO.26.3.036006]
\end{abstract}

Keywords: photoacoustics; radial artery; red blood cell aggregation; oxygen saturation; age.

Paper 200377R received Nov. 16, 2020; accepted for publication Mar. 2, 2021; published online Mar. 23, 2021.

\section{Introduction}

Red blood cells (RBCs) play an important, physiologically significant role in the human body, affecting hemodynamics as well as governing an oxygen transport. The oxygen-carrying capacity of RBCs is driven by the partial pressure of oxygen in blood vessels and the tissues they supply and is systemically assessed through metrics of oxygen saturation $\left(\mathrm{sO}_{2}\right) .{ }^{1}$ The hemodynamic aspects of RBCs, namely their flow profiles, pressures, and velocities, across the cardiovascular system play an equally important physiological role. ${ }^{2}$ Pathophysiological changes in the hemodynamic behavior of RBCs affect a wide variety of disorders. ${ }^{3}$ A phenomenon that is affected by the hemodynamic behaviors of RBCs is their aggregability, defined as the ability of the cells to form rouleaux in the presence of plasma proteins. ${ }^{4}$ This naturally occurring

*Address all correspondence to Michael C. Kolios, mkolios@ryerson.ca 
phenomenon is attributed to the cyclical variation of the shear forces forming when blood velocity gradients change during flow. ${ }^{5}$ It is thus affected by the hemodynamic behavior of vessels ${ }^{6}$ as well as RBC membrane factors that impact the electrostatic and steric interactions between cells. $^{7}$ The clinical significance of RBC aggregation becomes apparent in conditions where hyperaggregability has been reported. These include myocardial infarctions, bacterial infections, type 2 diabetes, and sickle cell disease. ${ }^{5}$ The study of RBC aggregation as a hemodynamic phenomenon has provided invaluable insights into the biophysical properties of this process. ${ }^{8,9}$ However, measurements of the $\mathrm{sO}_{2}$ in the presence of $\mathrm{RBC}$ aggregation have not been possible, impeding the assessment of the significance aggregation to the process of oxygen delivery.

The $\mathrm{SO}_{2}$ is universally recognized as one of the vital signs in medicine along with pulse rate, respiratory rate, temperature, and blood pressure. ${ }^{10,11}$ This metric measures the percentage of hemoglobin binding sites that are bound with oxygen in RBCs in the blood. Oxygen metabolism is tightly regulated in the body because hypoxemia (abnormally low blood oxygen level) can lead to acute adverse effects in several organ systems. ${ }^{10,12-15}$ Recently, the decreased $\mathrm{sO}_{2}$ of COVID-19 patients measured at hospital admission was reported as one of the strongest predictors of critical illness, with silent hypoxia concurrently occurring with other symptoms such as shortness of breath. ${ }^{16-18}$

In general, clinical $\mathrm{sO}_{2}$ measurements are conducted using a pulse oximeter that attaches at the tip of the patient's finger and is based on optical spectral differences between oxyhemoglobin $(\mathrm{HbO})$ and deoxyhemoglobin $(\mathrm{HbD})$. Pulse oximeters produce a measure of oxygenation which is sensitive only to the arterial vessels in the finger, not the veins. Another relevant clinical measurement is the arterial $\mathrm{sO}_{2}$, or " $\mathrm{SaO}_{2}$," a metric that is assessed using invasive blood draws from the radial artery followed by co-oximetry or blood gas analysis or indwelling catheter probes. ${ }^{19}$ However, in cases when peripheral perfusion is poor, as in states of hypovolemia, hypothermia, vasoconstriction, low cardiac output, and low mean arterial pressure, pulse oximeter readings become unreliable. ${ }^{20}$ Moreover, a variety of other clinical conditions such as vaso-occlusive crises in sickle cell anemia, severe anemia, methemoglobinemia, sepsis, or septic shock can influence the accuracy of pulse oximeter readings. ${ }^{19}$ Imaging techniques such as blood oxygen level-dependent magnetic resonance imaging (BOLD MRI), ${ }^{21}$ positron emission tomography (PET) ${ }^{22}$ or diffuse optical tomography or optical spectroscopy ${ }^{23,24}$ can assess the tissue oxygenation in vivo. Still, their millimeter-scale spatial resolutions limit their ability to assess the blood $\mathrm{sO}_{2}$ directly. In particular, BOLD MRI signals measure only $\mathrm{HbD}$ changes ${ }^{25}$ which are likely independent of the blood $\mathrm{sO}_{2}$ while PET uses ionizing radiation. Furthermore, these techniques are not portable and cannot be used to monitor $\mathrm{sO}_{2}$ in a clinical setting or in a point of care setting. For the noninvasive measurement of $\mathrm{sO}_{2}$ in more centrally located vessels, near-infrared spectroscopy (NIRS)-based oximetry is widely used. ${ }^{26}$ However, NIRS-based tissue oximeter cannot be used in a simultaneous assessment of the relationship between the $\mathrm{sO}_{2}$ and blood dynamics.

Photoacoustic (PA) imaging is an emerging technique in biomedical optics. It can provide not only anatomical structure but also functional information, complementing conventional ultrasound (US) imaging. Also, it provides greater penetration depth than conventional optical imaging methods since it relies on the detection of acoustic waves rather than ballistic photons. ${ }^{27,28}$ The high absorbance of hemoglobin within RBCs and the oxygen-dependent absorption of hemoglobin allows $\mathrm{PA}$ imaging to probe the microvasculature $\mathrm{sO}_{2}$, in vitro, as well as in vivo. ${ }^{29-31} \mathrm{PA}$ imaging is capable of noninvasive imaging of blood flow as well as simultaneous assessment of $\mathrm{sO}_{2}$, being utilized as the state-of-the-art tool for clinical applications such as investigation on the correlation between tissue characterization and oximetry.

However, no $\mathrm{PA} \mathrm{sO}_{2}$ studies have ever examined the impact of blood's hemodynamic behavior in the estimation of this important parameter. There are several methods to assess RBC aggregation, such as erythrocyte sedimentation rate, centrifugation methods, microscopic methods, low shear viscometry, US imaging, and analysis of light transmission (or reflection) of RBC suspension. ${ }^{32}$ The noninvasive measurement of RBC aggregation has been widely investigated using US imaging. ${ }^{33-35}$ RBC aggregation has also been used as a clinical biomarker for inflammation monitoring among other pathologies. ${ }^{8,9,36}$ However, US imaging probes the structure of RBC aggregates and does not directly measure physiological function of RBCs. As such, the assessment of both $\mathrm{sO}_{2}$ and hemodynamic behavior under blood flow needs to be independently 
assessed using noninvasive biomedical imaging using conventional optics ${ }^{37-40}$ and $\mathrm{US}^{33-35}$ respectively, but not in the same setting. Our group has reported on in vitro measurements that show the correlation between the $\mathrm{sO}_{2}$ and $\mathrm{RBC}$ aggregation using PA spectroscopy in static and flow conditions. ${ }^{41-43}$ Through carefully controlled in vitro conditions, we demonstrated that during a pulsatile cycle in a simulated pulsatile blood flow, the PA amplitude increased with decreasing flow velocity and decreased with increasing velocity. These changes in PA amplitude were due to RBC aggregation during small velocities and disaggregation during increased velocities and consistent with the findings widely reported using conventional US methodology. ${ }^{33,35}$ In addition to $\mathrm{RBC}$ aggregation, the PA-based $\mathrm{sO}_{2}$ also cyclically changed in-phase with $\mathrm{RBC}$ aggregation. These results suggest that $\mathrm{RBC}$ aggregates inhibited $\mathrm{O}_{2}$ release. In this paper, we describe the first in vivo evidence for the RBC aggregation-induced alterations in the $\mathrm{sO}_{2}$ measured in the radial artery of healthy subjects. Furthermore, a complex age-dependent correlation between the $\mathrm{sO}_{2}$ and $\mathrm{RBC}$ is discussed.

\section{Materials and Methods}

\subsection{Subject Recruitment and PA Imaging Protocol}

The human subject study was approved by the Research Ethic Board of Ryerson University (REB 2017-040). Healthy subjects were recruited based on the inclusion criteria listed in Table 1.

The subject sat down on a chair and immersed their left arm in a degassed warm $\left(36^{\circ} \mathrm{C}\right)$ water bath (Fig. 1). PA imaging was performed with a linear-array probe equipped PA imaging system (Vevo LAZR; LZ250-21 MHz of central frequency, 13 to $24 \mathrm{MHz}$ of bandwidth and 256 elements, FUJIFILM Visualsonics, Toronto, Ontario, Canada $)^{44}$ at the near-infrared wavelength $(700,750,800,850$, and $900 \mathrm{~nm})$. The pulsed-wave Doppler velocity $(V)$ was measured to locate the radial artery, and the measurement system was then switched to the PA imaging mode. A PA B-mode image was acquired to measure the pulsatile blood flow in the radial artery for $10 \mathrm{~s}$ at each optical wavelength $(\lambda)$. The total time that subjects' arms were immersed in water was $<5 \mathrm{~min}$. The time trace was acquired for each single wavelength then time-shifted to create a combined multispectral dataset in postprocessing. The detailed methods were described in our previous study. ${ }^{42,45}$

Table 1 Subject criteria and information.

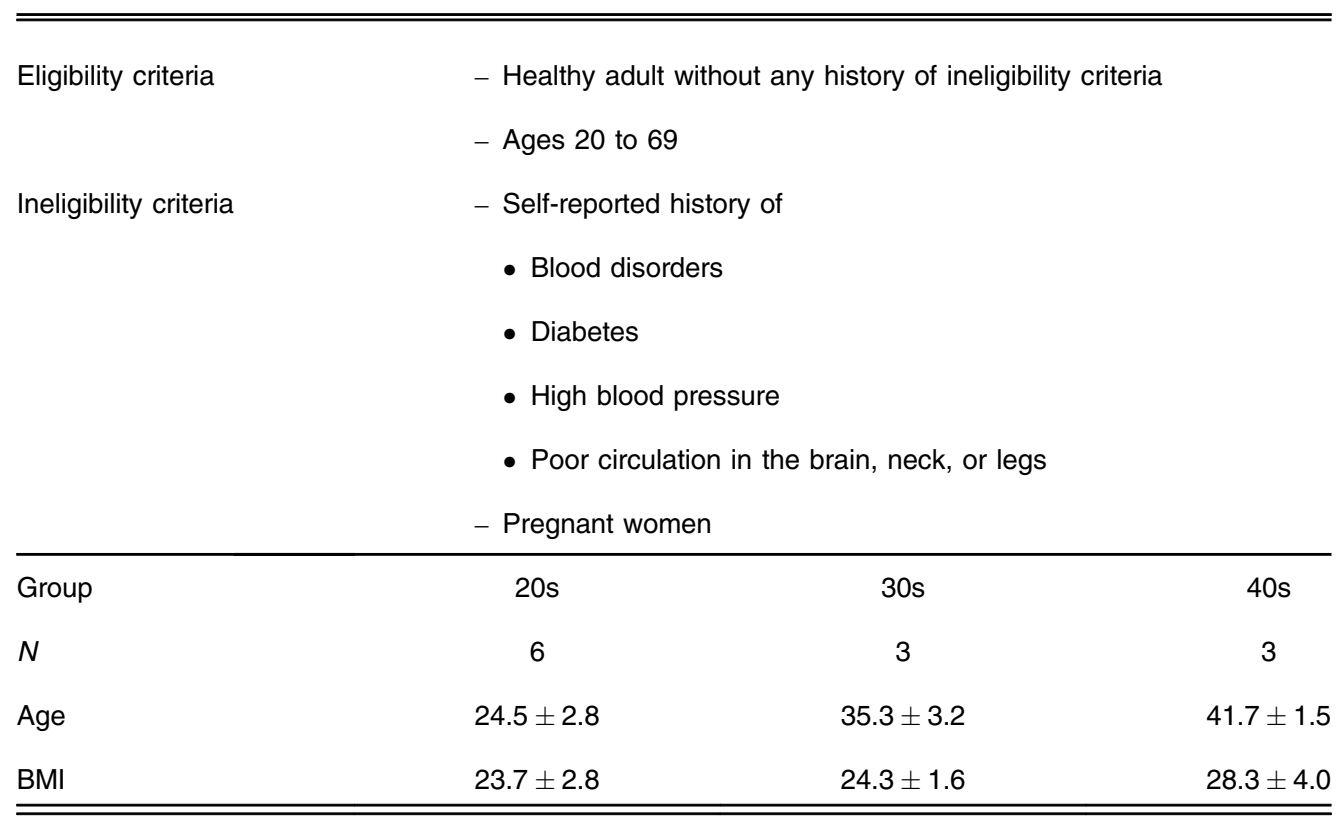

Note: $N$ : number of subjects, BMl: body mass index $\left(\mathrm{kg} / \mathrm{m}^{2}\right)$. 


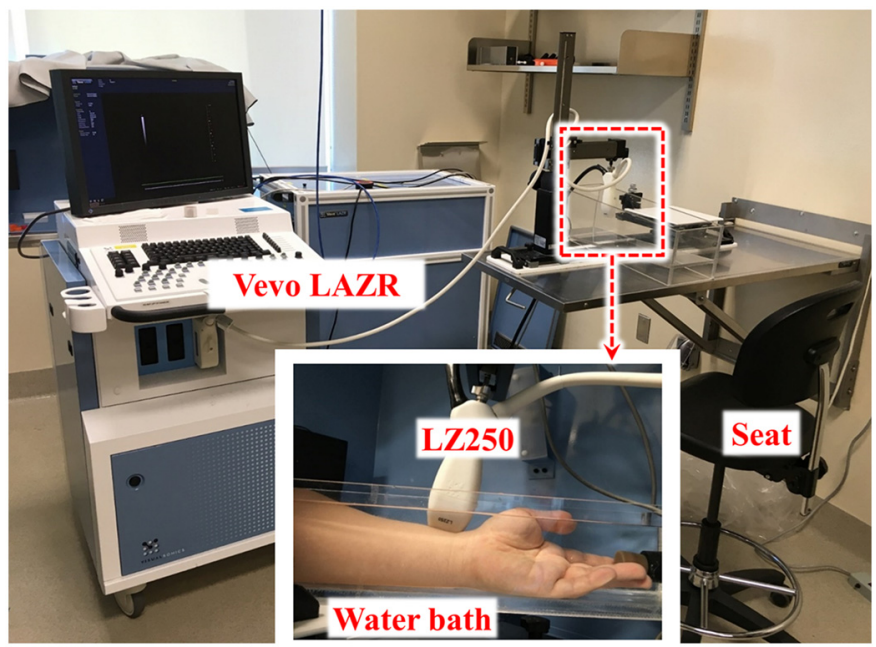

Fig. 1 Photograph of PA imaging system and a PA probe positioned over the region of the interest close to the wrist of a subject.

\subsection{Data Acquisition and Postprocessing: V, $\mathrm{Pa}$, and $\mathrm{sO}_{2}$}

A representative coregistered US (gray scale) and PA (color scale) image of the radial artery was shown in Fig. 2(a). The second quarter of the full field of view was chosen as a region of interest (ROI) to maximize the PA image acquisition rate to $20 \mathrm{~Hz}$. To avoid boundary-buildup or edge detection artifact from the upper and lower vessel walls, the upper and lower boundaries of the ROI were chosen at $10 \%$ margin within the vessel lumen. In the coregistered US image, the upper and lower vessel walls were tracked at each frame. For each wavelength and each subject, 200 frames of US images (20 frame/s) were analyzed to track the vessel wall. From the upper and lower walls tracked, the upper and lower boundaries for the ROI were computed by $10 \%$ margin within the vessel lumen. For example, if the locations of two walls were at 1 and $2 \mathrm{~mm}$ in

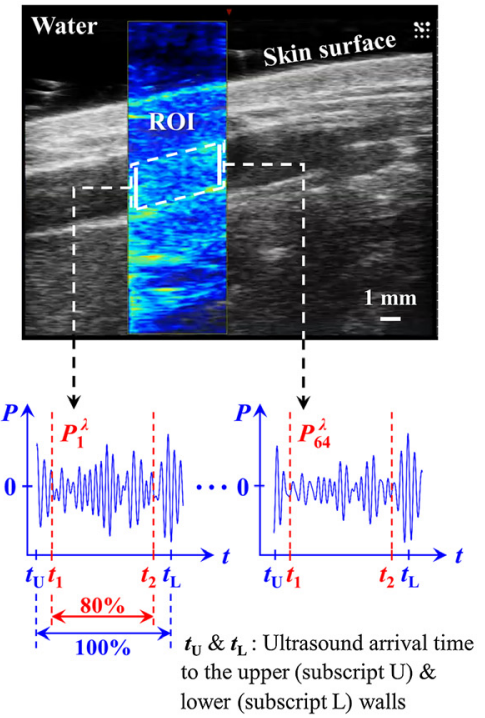

(a)

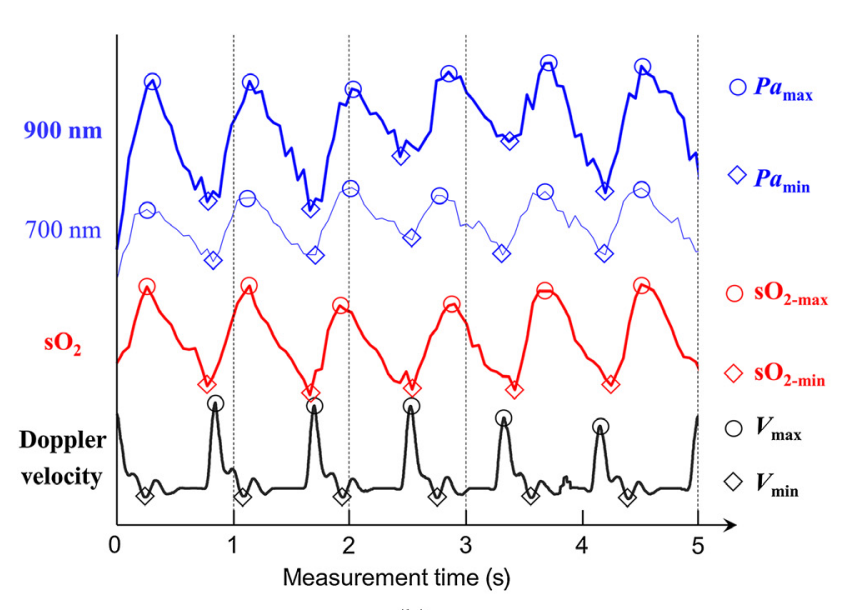

(b)

Fig. 2 (a) Representative US (gray scale) and PA, PA (color scale) image of the radial artery. The PA RF power $(\mathrm{Pa})$ from the ROI was computed by taking an average of the root-mean-square of each RF signal for each optical wavelength $(\lambda)$. (b) Representatives of $\mathrm{Pa}(900-\mathrm{nm}$-thick blue and 700-nm-thin blue), oxygen saturation $\left(\mathrm{sO}_{2}\right.$, red), and the Doppler velocity ( $V$, black). The subscripts max and min represent the maximum (denoted as a circle) and minimum (denoted as a diamond) value for each parameter. 
depth (corresponding to $t_{\mathrm{U}}$ and $t_{\mathrm{L}}$, respectively), the locations of two boundaries of ROI were 1.1 and $1.9 \mathrm{~mm}$ in depth (corresponding to $t_{1}$ and $t_{2}$, respectively) as shown in Fig. 2(a). The horizontal width of the ROI was fixed as a second quarter of the full field of view, whereas the vertical length (related to the vessel diameter) of the ROI was dependent on each frame. The VevoLAZR PA imaging system provides access to the prebeamformed RF data for all 256 transducer elements. These data are then beamformed postacquisition, and the amplitude of each signal is used to reconstruct the PA images shown in Fig. 2(a). The ROI is then selected from this reconstructed PA image. From beamformed radiofrequency (RF) PA signals (64 out of 256 elements) in the ROI, the PA power ( $\mathrm{Pa}$ ) was computed by taking an average of the rootmean-square of each RF signal for each $\lambda$, and addressed by

$$
P a(\lambda)=\frac{1}{64} \sum_{n=1}^{64} \frac{1}{E(\lambda)} \sqrt{\frac{1}{\Delta t} \int_{t_{1}}^{t_{2}}\left|P_{n}^{\lambda}(t)\right|^{2} \mathrm{~d} t},
$$

where $E$ is the laser energy in $\mathrm{J}$, which measured by Vevo LAZR, $\Delta t=t_{2}-t_{1}, t_{1}$ and $t_{2}$ represent the time in the RF signal, corresponding to the upper and lower boundaries of the ROI, respectively, as shown in Fig. 2(a), P is PA amplitude in voltage, $n$ is the element number of US detector. The velocity $(V)$ of the pulsatile blood flow measured for $10 \mathrm{~s}$. The number of pulsatile cycles was dependent on the subject (ranging from 11 to 15 cycles during the 10-s measurement for all subjects). The $\mathrm{sO}_{2}$ was estimated using the optical absorption at two applied wavelengths, which was derived from ${ }^{46}$

$$
\begin{gathered}
\mu_{a}(\lambda)=\varepsilon_{\mathrm{HbO}}(\lambda)[\mathrm{HbO}]+\varepsilon_{\mathrm{HbD}}(\lambda)[\mathrm{HbD}], \\
\mathrm{sO}_{2}=\frac{[\mathrm{HbO}]}{[\mathrm{HbO}]+[\mathrm{HbD}]}=\frac{\mu_{a}\left(\lambda_{2}\right) \varepsilon_{\mathrm{HbD}}\left(\lambda_{1}\right)-\mu_{a}\left(\lambda_{1}\right) \varepsilon_{\mathrm{HbD}}\left(\lambda_{2}\right)}{\mu_{a}\left(\lambda_{1}\right) \Delta \varepsilon_{\mathrm{Hb}}\left(\lambda_{2}\right)-\mu_{a}\left(\lambda_{2}\right) \Delta \varepsilon_{\mathrm{Hb}}\left(\lambda_{1}\right)},
\end{gathered}
$$

where $\mu_{a}$ is the absorption coefficient in $/ \mathrm{cm} ; \varepsilon_{\mathrm{HbO}}$ and $\varepsilon_{\mathrm{HbD}}$ are the known molar extinction coefficients of oxygenated hemoglobin ( $\mathrm{HbO})$ and deoxygenated hemoglobin $(\mathrm{HbD})$, respectively, in $/ \mathrm{cm} / M ; \Delta \varepsilon_{\mathrm{Hb}}=\varepsilon_{\mathrm{HbO}}-\varepsilon_{\mathrm{HbD}} ;[\mathrm{HbO}]$ and $[\mathrm{HbD}]$ are the molar concentrations of $\mathrm{HbO}$ and $\mathrm{HbD}$, respectively, in $M ; \lambda_{1}$ and $\lambda_{2}$ are two different $\lambda$ (700 and $900 \mathrm{~nm}$ in this study). $P$ can hence substitute for $\mu_{a} . P$ measured at 700 and $900 \mathrm{~nm}$ were synchronized to calculate the $\mathrm{sO}_{2}$ using Eq. (2), as shown in Fig. 2(b). $P$ was acquired at each of the wavelengths for $10 \mathrm{~s}$, and then the wavelength was switched to the next one. The time difference between acquisition at wavelengths was $20 \mathrm{~s}$, which is $10 \mathrm{~s}$ for wavelength switching and $10 \mathrm{~s}$ for acquisition at the wavelength. The $\mathrm{sO}_{2}$ assessment using our technique has been validated in previous studies ${ }^{47}$ and independently validated in pre- or clinical studies. ${ }^{48}$ Since the position and geometry of illuminated area were the same, the fluence effect could be minimized. Also, the effect of wavelength on fluence was somewhat compensated by using normalization to the energy at each wavelength. The representatives of $\mathrm{Pa}$, oxygen saturation $\left(\mathrm{sO}_{2}\right.$, red), and the Doppler velocity $(V$, black) were shown in Fig. 2(b).

The minimum $\left(\mathrm{Pa}_{\min }\right.$ and $\left.\mathrm{sO}_{2-\min }\right)$ and maximum $\left(\mathrm{Pa}_{\max }\right.$ and $\left.\mathrm{sO}_{2-\max }\right)$ values of $\mathrm{Pa}$ and $\mathrm{sO}_{2}$ were, respectively, averaged for $10 \mathrm{~s}$ to compare the variation in $\mathrm{Pa}$ and $\mathrm{sO}_{2}$ during the pulsatile blood flow as a function of $\lambda$ and age. Two sample $t$-test was conducted using built-in MALTAB function "ttest2.m," in terms of the $\mathrm{sO}_{2}$ and $\mathrm{Pa}$ changes versus age group.

\section{Results}

\subsection{PA Power versus Optical Wavelength}

The PA magnitude was dependent on the optical $\lambda$ as well as the study subjects. To compare this parameter, the $P a_{\max }$ and $P a_{\min }$ for all $\lambda$ were normalized to the $P a_{\min }$ at an isosbestic point $800 \mathrm{~nm}$ (the point at which $\mathrm{HbO}$ and $\mathrm{HbD}$ have the same optical absorption) for each subject. The $P a$ dependence on the $\lambda$ for each subject age group is shown in Fig. 3(a). The $P a_{\min }$ at $800 \mathrm{~nm}$ was set to $0 \mathrm{~dB}$ as a reference [green circles in Fig. 3(a)]. Both $P a_{\max }$ and $P a_{\min }$ increased with $\lambda$ for all groups, as shown in Fig. 3(a). In addition, the difference between 


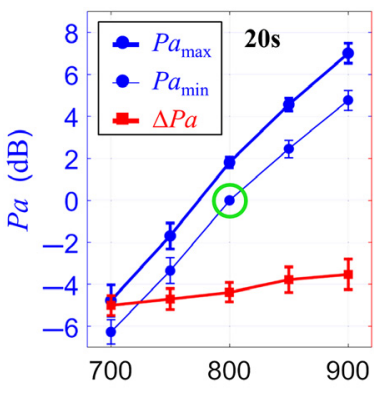

Optical wavelength $(\mathrm{nm})$

(a)

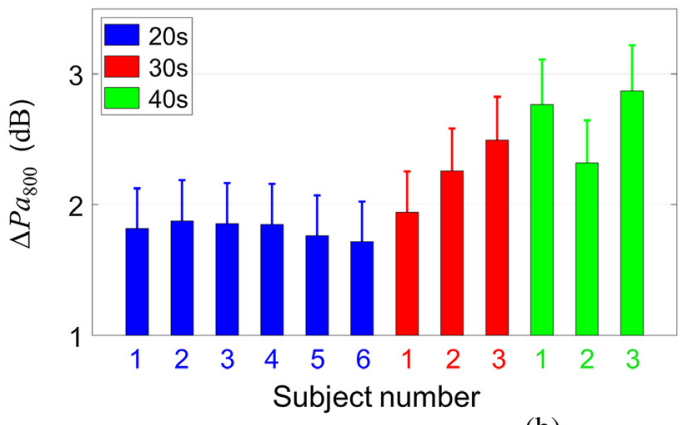

(b)
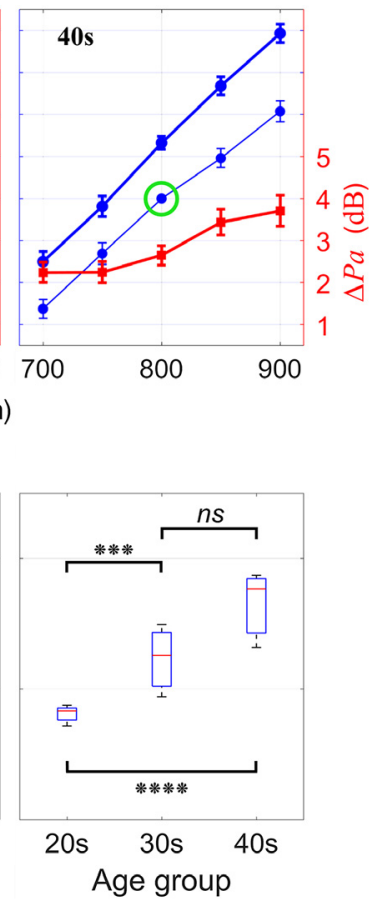

Age group

Fig. 3 (a) PA power $(P a)$ as a function of optical wavelength for each group of age 20s, 30s, and 40s. The thick and thin blue lines represent $P a_{\max }$ and $P a_{\min }$, respectively. The thick red line represents the variation from $P a_{\min }$ to $P a_{\max }(\Delta P a)$. The green circle indicates the isosbestic point ( $\mathrm{HbO}$ and $\mathrm{HbD}$ have the same optical absorption). The error bars represent the standard deviation from the number of cycles of the pulsatile flow for all subjects, i.e., 75, 39, and 38 cycles for each age group, respectively. (b) The $P a$ difference between $P a_{\min }$ and $P a_{\max }$ at an isosbestic point $800 \mathrm{~nm}\left(\triangle P a_{800}\right)$ for all subjects and the corresponding box and whisker plot (based on the average value of $\Delta P a_{800}$ for each subject) for each age group. The error bars represent the standard deviation from the number of cycles of the pulsatile flow for each subject. $n s$ : not significant, ${ }^{\star \star \star}$ : $p \leq 0.001{ }^{\star \star \star \star}: p \leq 0.0001$.

$P a_{\text {min }}$ and $P a_{\max }(\Delta P a)$ increased with the $\lambda$. The average values of $P a_{\max }, P a_{\min }$, and $\Delta P a$ at 700,800 , and $900 \mathrm{~nm}$ for all groups were tabulated (Table 2). The $P a$ difference between $P a_{\text {min }}$ and $P a_{\text {max }}$ at $800 \mathrm{~nm}\left(\Delta P a_{800}\right)$ for all subjects is shown in Fig. 3(b)-left. The error bars represent the standard deviation from the number of cycles of the pulsatile blood flow for each subject. The box and whisker plot based on the average values of $\Delta P a_{800}$ of each subject for age groups is shown in Fig. 3(b)-right. The $\Delta P a_{800}$ increased with age, i.e., 1.8, 2.2, and $2.7 \mathrm{~dB}$ for the age group of subjects in their $20 \mathrm{~s}, 30 \mathrm{~s}$, and $40 \mathrm{~s}$, respectively.

Table 2 The values of minimum $\left(P a_{\min }\right)$ and maximum $\left(P a_{\max }\right)$ of PA power and the difference between $P a_{\min }$ and $P a_{\min }(\Delta P a)$ at 700,800 , and $900 \mathrm{~nm}$ for all groups. The error bars represent the standard deviation from the number of cycles of the pulsatile flow for all subjects.

\begin{tabular}{|c|c|c|c|c|c|c|c|c|c|}
\hline \multirow{2}{*}{$\frac{\text { Group }}{\lambda(\mathrm{nm})}$} & \multicolumn{3}{|c|}{$20 s$} & \multicolumn{3}{|c|}{$30 \mathrm{~s}$} & \multicolumn{3}{|c|}{$40 \mathrm{~s}$} \\
\hline & 700 & 800 & 900 & 700 & 800 & 900 & 700 & 800 & 900 \\
\hline$P a_{\max }(\mathrm{dB})$ & $-4.8 \pm 0.7$ & $1.8 \pm 0.3$ & $7.0 \pm 0.5$ & $-3.8 \pm 0.6$ & $2.2 \pm 0.4$ & $7.3 \pm 0.6$ & $-3.0 \pm 0.5$ & $2.7 \pm 0.3$ & $7.9 \pm 0.4$ \\
\hline$P a_{\min }(\mathrm{dB})$ & $-6.3 \pm 0.6$ & 0 & $4.8 \pm 0.5$ & $-5.6 \pm 0.6$ & 0 & $4.4 \pm 0.6$ & $-5.2 \pm 0.5$ & 0 & $4.2 \pm 0.5$ \\
\hline$\Delta P a(\mathrm{~dB})$ & $1.5 \pm 0.2$ & $1.8 \pm 0.3$ & $2.2 \pm 0.4$ & $1.8 \pm 0.2$ & $2.2 \pm 0.4$ & $2.9 \pm 0.4$ & $2.2 \pm 0.2$ & $2.7 \pm 0.3$ & $3.7 \pm 0.4$ \\
\hline
\end{tabular}


Bok, Hysi, and Kolios: In vivo photoacoustic assessment of the oxygen saturation changes...

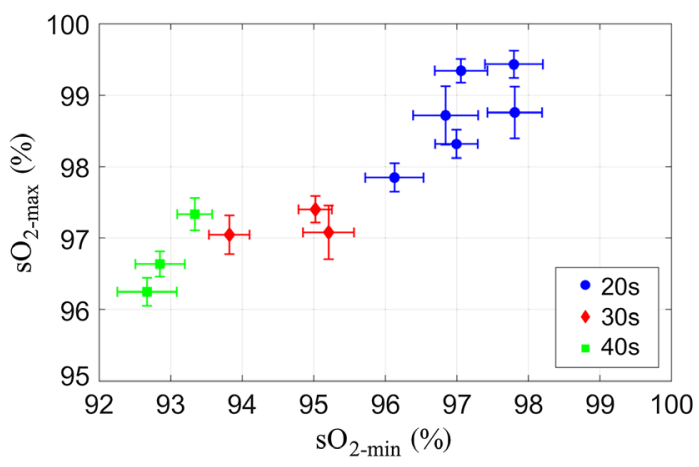

Fig. 4 Oxygen saturation $\left(\mathrm{sO}_{2}\right)$ for all subjects-subjects in their 20s (blue dots), subjects in their 30 s (red diamonds), and subjects in their 40 s (green square). The $\mathrm{sO}_{2}$ was computed for $P a_{\max }$ $\left(\mathrm{sO}_{2-\max }\right)$ and $\mathrm{Pa}_{\min }\left(\mathrm{sO}_{2-\min }\right)$, respectively. The error bars represent the standard deviation from the number of cycles of the pulsatile flow.

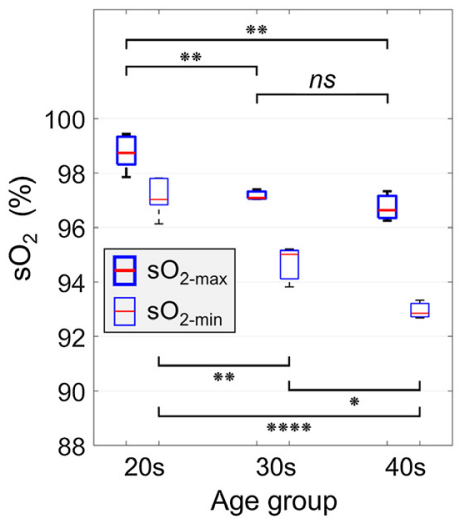

(a)

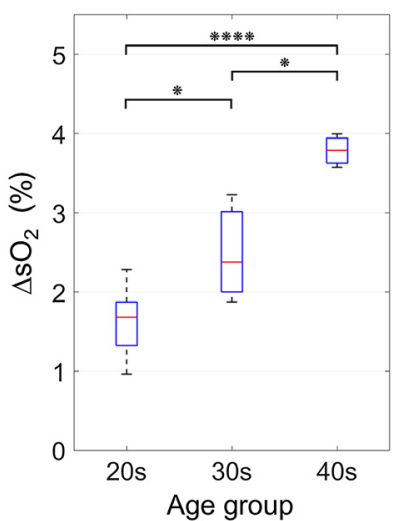

(b)

Fig. 5 (a) Oxygen saturation $\left(\mathrm{sO}_{2}\right)$ for each group based on the average value of $\mathrm{sO}_{2}$ of each subject. The thick $\left(\mathrm{sO}_{2-\max }\right)$ and thin $\left(\mathrm{sO}_{2-\min }\right)$ symbols represent the $\mathrm{sO}_{2}$ computed using the two wavelength $(700$ and $900 \mathrm{~nm})$ methods at $P a_{\max }$ and $P a_{\min }$, respectively. (b) The difference between $\mathrm{sO}_{2-\max }$ and $\mathrm{sO}_{2-\min }\left(\Delta \mathrm{sO}_{2}\right)$ for each group. ns: not signigicant, ${ }^{*}: p \leq 0.05,{ }^{*}: p \leq 0.01$, ${ }^{* * * *}: p \leq 0.0001$.

\section{$3.2 \mathrm{SO}_{2}$ versus Subject Age}

The $\mathrm{sO}_{2}$ estimated from $P a_{\min }\left(\mathrm{sO}_{2-\min }\right)$ and $P a_{\max }\left(\mathrm{sO}_{2-\max }\right)$ for each group of age $20 \mathrm{~s}, 30 \mathrm{~s}$, and 40s were shown in Fig. 4, respectively. The average $\mathrm{sO}_{2-\max }$ for subjects in their $20 \mathrm{~s}$, 30s, and 40s were $98.7 \%, 97.2 \%$, and $96.7 \%$, respectively [Fig. 5(a)]. This indicates that the difference in $\mathrm{sO}_{2 \text {-max }}$ between the youngest and oldest subjects was $2.0 \%(p \leq 0.01)$. On the other hand, the average $\mathrm{sO}_{2 \text {-min }}$ for subjects in their $20 \mathrm{~s}, 30 \mathrm{~s}$, and $40 \mathrm{~s}$ were $97.1 \%, 94.7 \%$, and $93.0 \%$, respectively [Fig. 5(a)]. The $\mathrm{sO}_{2-\min }$ difference between the $20 \mathrm{~s}$ and $40 \mathrm{~s}$ groups was $4.1 \%$ $(p \leq 0.0001)$. The $\mathrm{sO}_{2}$ difference between $\mathrm{sO}_{2-\min }$ and $\mathrm{sO}_{2-\max }\left(\Delta \mathrm{sO}_{2}\right)$ during a pulsatile cycle increased with age, i.e., $1.6 \%, 2.5 \%$, and $3.8 \%$ for the age group of subjects in their $20 \mathrm{~s}, 30 \mathrm{~s}$, and 40s, respectively [Fig. 5(b)].

\section{Discussion}

Several studies have demonstrated the PA assessment of the $\mathrm{sO}_{2} \cdot{ }^{28,46,49,50}$ However, the complexity of hemodynamic and/or hemorheological behaviors such as RBC aggregation/disaggregation and deformation have not been considered as a possible factor that could alter the estimated $\mathrm{sO}_{2}$. 
Our group has studied the feasibility of using PA spectroscopy for assessing the relation between $\mathrm{RBC}$ aggregation and $\mathrm{sO}_{2}$ under pulsatile blood flow in vitro using experimental and theoretical methods. ${ }^{41,42}$ This paper describes the first-in-human feasibility study on the dependence of the $\mathrm{sO}_{2}$ on the pulsatile blood flow conditions of the radial artery. The experimental results show an age dependence of the $\mathrm{sO}_{2}$ measurements in vivo.

\subsection{PA Power versus Age - Aggregability of RBCs}

Several studies reported on the correlation between RBC aggregation and age. ${ }^{51-53}$ Woodward et al. ${ }^{51}$ measured hemorheological variables (blood viscosity, RBC aggregation, and fibrinogen) from subjects ( 25 to 74 years old) in association with cardiovascular risk factors. They found that RBC aggregation and fibrinogen increased with age. Christy et al. ${ }^{52}$ reported a significant increase in RBC aggregation with age (20 to 59 years old), establishing a significant correlation between phagocytic activity and RBC aggregability. According to Simmonds et al. ${ }^{53}$ the mechanism of the age-related increase in RBC aggregation is that aging decreased the electrostatic repulsive forces between cell surfaces, thus promoting RBC aggregation alongside with increased plasma fibrinogen concentration.

During pulsatile blood flow, the dominant hemorheological characteristic is the cyclical aggregation and disaggregation phases of RBCs. ${ }^{5,32}$ During systole, the blood flow velocity is maximum, generating maximal shear rate within vessels, and as a result, the RBCs in the radial artery flow as single cells. During diastole, on the other hand, the blood flow velocity is at a minimum, resulting in a minimal shear rate, leading to the formation of RBCs rouleaux. ${ }^{5,32}$ In PA imaging, the RBC aggregates form a larger effective absorber compared to single cells, increasing the $P a$ signal. ${ }^{41,42,54,55}$ In Fig. 2(b), the $P a$ and Doppler velocity $V$ were out of phase during a systolic-diastolic cycle. A higher $P a$ is expected in the presence of aggregates and a lower $P a$ from single RBCs. This occurred at all wavelengths of illumination, and as we have shown in earlier studies, it can approximate the aggregate size. ${ }^{41}$

The PA amplitude is a function of $\mu_{a}$ (including the absorption cross-section) and an absorber size. According to Eq. (1), $\mu_{a}$ can be represented as a linear combination of $\varepsilon_{\mathrm{HbO}}, \varepsilon_{\mathrm{HbD}}$, [HbO] and [HbD], depending on $\lambda$. The $\mathrm{sO}_{2}$ can be derived by combining $\mu_{a}$ at two wavelengths as addressed in Eq. (2). Given the $\mathrm{sO}_{2}$ also varies with the absorber size affecting the $\mu_{a}$, the $P a$ must have a nonlinear relation with the absorber size. However, the $P a$ at an isosbestic point $800 \mathrm{~nm}$ is not dependent on the $\mathrm{sO}_{2}$, resulting in a linear relation between the $\mathrm{Pa}$ and the absorber size, as previously demonstrated by our group. ${ }^{41}$ The $\Delta P a_{800}$ increased with age [Fig. 3(b)], suggesting that the RBC aggregability also increased with age (since the PA signal is a surrogate metric of the aggregate size).

\subsection{Effect of RBC Aggregation on PA Power as a Function of Absorber Size and $\mathrm{SO}_{2}$}

None of the abovementioned studies have examined the impact of RBC aggregation on the blood $\mathrm{sO}_{2}$ measurements in humans. It is well known that $\mathrm{RBC}$ aggregation and plasma viscosity play an important role in determining the overall blood viscosity. ${ }^{56}$ Realizing the fundamental function of RBCs in oxygen transport, Tateishi et al. ${ }^{57,58}$ were the first to postulate on the correlation between RBC aggregation and $\mathrm{O}_{2}$ release from erythrocytes and its impact on the $\mathrm{sO}_{2} \cdot{ }^{59} \mathrm{In}$ addition, the relation between $\mathrm{RBC}$ aggregation and the $\mathrm{sO}_{2}$ was theoretically and experimentally investigated through in vitro PA imaging by our group. ${ }^{41}$ Similar to the Tateishi's findings and our in vitro experiments, the in vivo results in this work demonstrate the impact of RBC aggregation on the $\mathrm{sO}_{2}$ measurement.

The $P a$ increases due to two factors: an increase in the size of the absorber (single cells versus $\mathrm{RBC}$ aggregates) and the change in $\mathrm{sO}_{2}$. The average values of $P a$ as a function of $\lambda$ for the group of subjects in their 40s are shown in Fig. 6(a). The $P a_{\min }$ represents measurements when nonaggregated RBCs flow in the radial artery at maximal velocity (when the shear rates inside the radial artery are highest). RBC aggregation increases the $P a$ due to an increase in optical absorber size. Since the optical absorber size increases as RBCs aggregate, the $P a_{\min }$ increases by $\Delta P a_{800}(2.7 \mathrm{~dB}$ for subjects in their $40 \mathrm{~s})$ for all $\lambda\left(P a_{\min }+\Delta P a_{800}\right.$, depicted by the 


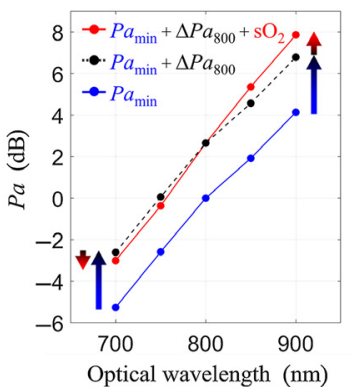

(a)

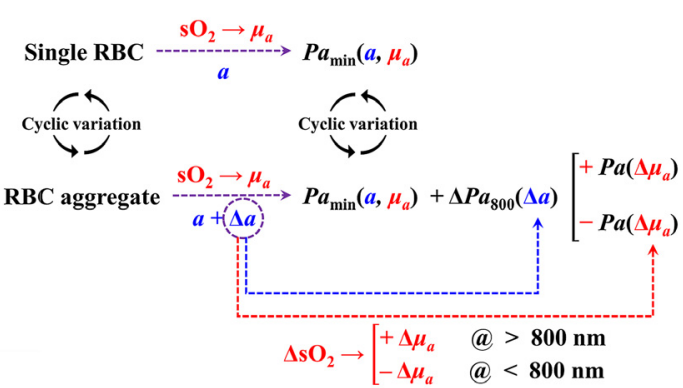

(b)

Fig. 6 (a) The effect of RBC aggregation on PA power $(P a)$ in relation to an optical absorber size and oxygen saturation $\left(\mathrm{sO}_{2}\right)$. (b) Schematic diagram of the effect of $\mathrm{RBC}$ aggregation on $\mathrm{Pa}$ in relation to the absorber size and the $\mathrm{sO}_{2}$. $a$ and $\Delta a$ represent the size of single $\mathrm{RBC}$ and the increased size due to aggregation, respectively.

blue-black arrow). This can be schematically represented by $\Delta P a_{800}(\Delta a)$ in Fig. 6(b). The second reason for the changes in the $\mathrm{Pa}$ is the change in the $\mathrm{sO}_{2}$ due to $\mathrm{RBC}$ aggregation. The difference between $P a_{\max }$ and " $P a_{\min }+\Delta P a_{800}$ " can be identified as the contribution to the change in $\mathrm{sO}_{2}$ caused by $\mathrm{RBC}$ aggregation to the overall $\mathrm{PA}$ signal, $\left(P a_{\min }+\right.$ $\left.\Delta P a_{800}+\mathrm{sO}_{2}=P a_{\max }\right)$. Combining Eqs. (1) and (2), the $\mu_{a}$ can be expressed as a function of $\mathrm{sO}_{2}$,

$$
\mu_{a}=\left(\left(\varepsilon_{\mathrm{HbO}}-\varepsilon_{\mathrm{HbD}}\right) \mathrm{sO}_{2}+\varepsilon_{\mathrm{HbD}}\right)([\mathrm{HbO}]+[\mathrm{HbD}])
$$

Since $\varepsilon_{\mathrm{HbO}}$ is larger than $\varepsilon_{\mathrm{HbD}}$ for $\lambda>800 \mathrm{~nm},{ }^{60}$ the $\mathrm{sO}_{2}$-induced increase in $\mu_{a}$ results in an increased $\mathrm{Pa}$ with $\mathrm{sO}_{2}$, as shown in Fig. 6. On the other hand, since $\varepsilon_{\mathrm{HbO}}$ is smaller than $\varepsilon_{\mathrm{HbD}}$ for $\lambda<800 \mathrm{~nm}$, the $\mathrm{sO}_{2}$-induced decrease in $\mu_{a}$ results in a decreased $P a$. This can be schematically denoted by $+P a\left(\Delta \mu_{a}\right)$ and $-P a\left(\Delta \mu_{a}\right)$ for $\lambda>800 \mathrm{~nm}$ and $\lambda<800 \mathrm{~nm}$, respectively, in Fig. 6(b). The in vivo experimental results demonstrating the effect of RBC aggregation on the $\mathrm{Pa}$ and how this depends on the $\mathrm{RBC} \mathrm{S}_{2}$ are also supported by our group's previous in vitro work mimicking the radial artery flow conditions. ${ }^{41}$ This interpretation applies to all age groups.

\subsection{Relationship between $\mathrm{sO}_{2}$ versus Age}

$P a_{\text {max }}$ could be derived from $P a_{\text {min }}$ by combining the effect of the increase in both absorber size and $\mathrm{sO}_{2}$ due to $\mathrm{RBC}$ aggregation. Hence, the $\mathrm{sO}_{2}$ computed from the $P a_{\max }\left(\mathrm{sO}_{2-\max }\right)$ were higher than that from the $P a_{\text {min }}\left(\mathrm{sO}_{2-\min }\right)$ for all groups [as shown in Fig. 5(a)]. In addition, both $\mathrm{sO}_{2-\max }$ and $\mathrm{sO}_{2-\text { min }}$ decreased with increasing age. It has been reported that older persons have a lower $\mathrm{sO}_{2}$ than their younger counterparts. ${ }^{61-65}$ Even though the direct relation between the $\mathrm{sO}_{2}$ and age has not been fully investigated, it was reported that dysphagia (swallowing difficulties) could mediate the relation between $\mathrm{sO}_{2}$ and age. ${ }^{65}$ Older people are more likely to experience dysphagia which impairs pulmonary function and lowers $\mathrm{sO}_{2}$. The present experimental results indicated that the $\mathrm{sO}_{2}$ decreases with age, despite the subject cohort comprising healthy individuals.

The difference between $\mathrm{sO}_{2-\max }$ and $\mathrm{sO}_{2-\min }\left(\Delta \mathrm{sO}_{2}\right)$ increased with age, as shown in Fig. 5(b). This phenomenon was consistent with the relation between $\Delta P a_{800}$ and age, as shown in Fig. 3(b). The $P a_{800}$ represents the effects of RBC aggregability, independent of $\mathrm{sO}_{2}$, which increased with age. Since oxygen release is inhibited by RBC aggregation, ${ }^{57,58}$ higher aggregability results in more hemoglobin molecules to be bound oxygen molecules. Despite a negative correlation between the $\mathrm{sO}_{2-\min }$ and age, the $\mathrm{RBC}$ aggregation-induced increase in the $\mathrm{sO}_{2}$ $\left(\Delta \mathrm{sO}_{2}\right)$ resulted in a positive correlation between $\Delta \mathrm{sO}_{2}$ and age. This is why the correlation between the $\mathrm{sO}_{2 \text {-min }}$ and age is steeper than the correlaiton between the $\mathrm{sO}_{2 \text {-max }}$ and age, as shown in Fig. 5(a). 


\subsection{Limitations of the Study}

The findings of this study suggest the feasibility of the PA assessment of both $\mathrm{sO}_{2}$ and its correlation to age, in vivo. However, there are limitations to this study that form the basis for future extension of this work. The study, whose recruitment is suspended during the global COVID-19 pandemic, enrolled a smaller than desired subject group and was mainly composed of male volunteers. The sex dependence on $\mathrm{Pa}$ related to the correlation between $\mathrm{sO}_{2}$ and age must be further investigated. The laser fluctuation which sometimes occur from pulse to pulse or from wavelength to wavelength should be measured and corrected. The absolute values for the $\mathrm{sO}_{2}$ presented in this paper have not been corrected for the effects of laser fluence. ${ }^{66}$ The radial artery depth from the skin surface is $\sim 5 \mathrm{~mm}$. As such, the effects of fluence (spectral coloring) ${ }^{67} \mathrm{might}$ not be as significant as it is in other PA applications. Specifically, in Eq. (3), the $\mathrm{sO}_{2}$ is calculated by a ratio of $\mu_{a}$. The PA amplitude $(P)$ is a function of $\mu_{a}$ and fluence $(\varphi)$, so that the $\mathrm{sO}_{2}$ can be represented by the ratio of " $P / \varphi$." Moreover, since the position and geometry were the same, variations in fluence are minimized. In addition, the normalization to the laser energy at each wavelength could also contribute to a reduction of the fluence effects. As such, the reported absolute values might not represent the true $\mathrm{sO}_{2}$ for each subject. In addition, this quantity is dependent on the subject's skin color (leading to a larger optical path length to the radial artery, requiring fluence correction). An additional limitation is that the upper limit of age group was in $40 \mathrm{~s}$. Further investigation for the older age groups (50s, 60, and 70s) should be conducted to study the age dependence further.

Despite these limitations, this study demonstrates the feasibility of the measurements and confirms previous in vitro findings. Future studies could be done using a portable probe, opening the potential for doing this investigation more easily. In addition, quantitative measurement of RBC aggregation by US such as the structure-factor-size-estimation ${ }^{68}$ should be further applied to this study to correlate both PA and US modalities.

\section{Conclusion}

In this study, the correlation between the $\mathrm{sO}_{2}$ and $\mathrm{RBC}$ aggregation under the pulsatile blood flow in the human radial artery, and its age dependence, was investigated using PA imaging. $\mathrm{RBC}$ aggregability increased with age, as observed by the age-induced increase in the $\triangle P a_{800}$ metric during $\mathrm{RBC}$ aggregation. The $\mathrm{sO}_{2}$ change during a cycle of pulsatile blood flow also increased with age, and it was attributed to the presence of increased RBC aggregation in older subjects. This study is the first study to examine how the in vivo changes in $\mathrm{sO}_{2}$ during blood flow in human body can be assessed using PA imaging. This study demonstrates the effect of $\mathrm{RBC}$ aggregation on the $\mathrm{sO}_{2}$ change during a cardiac cycle in healthy volunteers and the increase in the $\mathrm{sO}_{2}$ change with age. Although these preliminary observations were conducted in only 12 healthy participants, this work demonstrates the feasibility of the measurement in vivo. Such measurements might shed light on the clinical importance of the complex relation between the blood viscosity induced by $\mathrm{RBC}$ aggregation and the oxygen delivery related to $\mathrm{sO}_{2}$. As a noninvasive measurement, $\mathrm{PA}$ imaging of blood $\mathrm{sO}_{2}$ could be extended to the detection of blood pathologies that alter the viscosity, modifying flow behaviors and oxygen delivery.

\section{Disclosures}

The authors have no relevant financial interests in the manuscript and no other potential conflicts of interest to disclose.

\section{Acknowledgments}

This work was funded by the Natural Sciences and Engineering Research Council of Canada/ Canadian Institutes of Health Research - Collaborative Health Research Projects Grant No. 462315-2014 and an NSERC Discovery Grant (RGPIN-2017-06496). Funding to purchase the equipment was provided by the Canada Foundation for Innovation, the Ontario Ministry of 
Bok, Hysi, and Kolios: In vivo photoacoustic assessment of the oxygen saturation changes...

Research and Innovation, and Ryerson University. E. Hysi is supported by Banting and KRESCENT Fellowships by the Canadian Institutes of Health Research and the Kidney Foundation of Canada, respectively. We thank Elizabeth Berndl at the Department of Physics at Ryerson University for providing technical support.

\section{References}

1. M. L. Ellsworth, R. N. Pittman, and C. G. Ellis, "Measurement of hemoglobin oxygen saturation in capillaries," Am. J. Physiol. Circ. Physiol. 252(5), H1031-H1040 (1987).

2. T. W. Secomb, "Hemodynamics," Compr. Physiol. 6(2), 975-1003 (2016).

3. R. N. Mitchell, "Hemodynamic disorders thromboembolic disease and shock," in Robbins and Cotran Pathologic Basis of Disease, 9th ed., V. Kumar, A. K. Abbas, and J. C. Aster, Eds., pp. 113-136, Elsevier (2014).

4. G. Barshtein, R. Ben-Ami, and S. Yedgar, "Role of red blood cell flow behavior in hemodynamics and hemostasis," Expert Rev. Cardiovasc. Ther. 5(4), 743-752 (2007).

5. O. K. Baskurt, B. Neu, and H. J. Meiselman, Red Blood Cell Aggregation, CRC Press, Boca Raton, Florida (2011).

6. H. H. Lipowsky, "Microvascular rheology and hemodynamics," Microcirculation 12(1), 5-15 (2005).

7. S. Chien, "Biophysical behavior of red cells in suspensions," in The Red Blood Cell: Volume 2, 2nd ed., D. M. Surgenor, Ed., pp. 1031-1133, Academic Press, New York (1975).

8. F. T. H. Yu et al., "A local increase in red blood cell aggregation can trigger deep vein thrombosis: evidence based on quantitative cellular ultrasound imaging," J. Thromb. Haemost. 9(3), 481-488 (2011).

9. J. Tripette et al., "In vivo venous assessment of red blood cell aggregate sizes in diabetic patients with a quantitative cellular ultrasound imaging method: proof of concept," PLoS One 10(4), e 0124712 (2015).

10. D. W. Green and G. Kunst, "Cerebral oximetry and its role in adult cardiac, non-cardiac surgery and resuscitation from cardiac arrest," Anaesthesia 72(Suppl. 1), 48-57 (2017).

11. R. A. L. Walton and B. D. Hansen, "Venous oxygen saturation in critical illness," J. Vet. Emerg. Crit. Care 28(5), 387-397 (2018).

12. B. D. Kent, P. D. Mitchell, and W. T. Mcnicholas, "Hypoxemia in patients with COPD: cause, effects, and disease progression," Int. J. Chron. Obstruct. Pulmon. Dis. 6(1), 199-208 (2011).

13. M. R. Hanudel et al., "Effects of acute kidney injury and chronic hypoxemia on fibroblast growth factor 23 levels in pediatric cardiac surgery patients," Pediatr. Nephrol. 31(4), 661-669 (2016).

14. A. Puttappa et al., "Prolonged unexplained hypoxemia as initial presentation of cirrhosis: a case report," Am. J. Case Rep. 18, 1-6 (2017).

15. B. D. Kussman et al., "Cerebral oxygen saturation in children with congenital heart disease and chronic hypoxemia," Anesth. Analg. 125(1), 234-240 (2017).

16. A. Thomas, G. Haljan, and A. Mitra, "Lung ultrasound findings in a 64-year-old woman with COVID-19," Can. Med. Assoc. J. 192(15), E399 (2020).

17. R. G. Wilkerson et al., "Silent hypoxia: a harbinger of clinical deterioration in patients with COVID-19," Am. J. Emerg. Med. 38(10), 2243.e5-2243.e6 (2020).

18. M. Petrilli et al., "Factors associated with hospital admission and critical illness among 5279 people with coronavirus disease 2019 in New York City: prospective cohort study," Br. Med. J. 369, m1966 (2020).

19. E. D. Chan, M. M. Chan, and M. M. Chan, "Pulse oximetry: understanding its basic principles facilitates appreciation of its limitations," Respir. Med. 107(6), 789-799 (2013).

20. P. A. Kyriacou et al., "Investigation of oesophageal photoplethysmographic signals and blood oxygen saturation measurements in cardiothoracic surgery patients," Physiol. Meas. 23(3), 533-545 (2002).

21. T. Christen, D. S. Bolar, and G. Zaharchuk, "Imaging brain oxygenation with MRI using blood oxygenation approaches: methods, validation, and clinical applications," Am. J. Neuroradiol. 34(6), 1113-1123 (2013). 
Bok, Hysi, and Kolios: In vivo photoacoustic assessment of the oxygen saturation changes...

22. N. Fleming et al., "Imaging tumour hypoxia with positron emission tomography," Br. J. Cancer 112(2), 238-250 (2015).

23. Y. Hoshi and Y. Yamada, "Overview of diffuse optical tomography and its clinical applications,” J. Biomed. Opt. 21(9), 091312 (2016).

24. N. C. Biswal, Y. Xu, and Q. Zhu, "Imaging tumor oxyhemoglobin and deoxyhemoglobin concentrations with ultrasound-guided diffuse optical tomography," Technol. Cancer Res. Treat. 10(5), 417-429 (2011).

25. R. G. Evans et al., "Don't be so BOLD: potential limitations in the use of BOLD MRI for studies of renal oxygenation," Kidney Int. 71(12), 1327-1328 (2007).

26. P. B. Benni et al., "A validation method for near-infrared spectroscopy based tissue oximeters for cerebral and somatic tissue oxygen saturation measurements," J. Clin. Monit. Comput. 32(2), 269-284 (2018).

27. P. Beard, "Biomedical photoacoustic imaging," Interface Focus 1(4), 602-631 (2011).

28. M. Xu and L. V. Wang, "Photoacoustic imaging in biomedicine," Rev. Sci. Instrum. 77(4), 041101 (2006).

29. F. Cao et al., "Photoacoustic imaging in oxygen detection," Appl. Sci. 7(12), 1262 (2017).

30. S. Hu, "Emerging concepts in functional and molecular photoacoustic imaging," Curr. Opin. Chem. Biol. 33, 25-31 (2016).

31. S. Wang et al., "Recent advances in photoacoustic imaging for deep-tissue biomedical applications," Theranostics 6(13), 2394-2413 (2016).

32. O. K. Baskurt and H. J. Meiselman, "Erythrocyte aggregation: basic aspects and clinical importance," Clin. Hemorheol. Microcirc. 53(1-2), 23-37 (2013).

33. G. Cloutier and Z. Qin, "Ultrasound backscattering from non-aggregating and aggregating erythrocytes-a review," Biorheology 34(6), 443-470 (1997).

34. T.-H. Bok et al., "Feasibility study of high-frequency ultrasonic blood imaging in human radial artery," J. Med. Biol. Eng. 35(1), 21-27 (2015).

35. D.-G. Paeng, K.-H. Nam, and K. K. Shung, "Cyclic and radial variation of the echogenicity of blood in human carotid arteries observed by harmonic imaging," Ultrasound Med. Biol. 36(7), 1118-1124 (2010).

36. B. Chayer et al., "Pilot clinical study of quantitative ultrasound spectroscopy measurements of erythrocyte aggregation within superficial veins," Clin. Hemorheol. Microcirc. 74(2), 109-126 (2020).

37. M. J. R. Polson and G. L. Morris, "Method of measuring the oxygen saturation in pulsating blood flow," US5448991A (1995).

38. T. Aoyagi, "Pulse oximetry: its invention, theory, and future," J. Anesth. 17(4), 259-266 (2003).

39. E. Sinex, "Pulse oximetry: principles and limitations," Am. J. Emerg. Med. 17(1), 59-66 (1999).

40. E. Roussakis et al., "Oxygen-sensing methods in biomedicine from the macroscale to the microscale," Angew. Chemie Int. Ed. 54(29), 8340-8362 (2015).

41. T.-H. Bok, E. Hysi, and M. C. Kolios, "In vitro photoacoustic spectroscopy of pulsatile blood flow: probing the interrelationship between red blood cell aggregation and oxygen saturation," J. Biophotonics 11, e201700300 (2018).

42. T.-H. Bok, E. Hysi, and M. C. Kolios, "Simultaneous assessment of red blood cell aggregation and oxygen saturation under pulsatile flow using high-frequency photoacoustics," Biomed. Opt. Express 7(7), 2769-2780 (2016).

43. Y. X. Huang et al., "Human red blood cell aging: correlative changes in surface charge and cell properties,” J. Cell. Mol. Med. 15(12), 2634-2642 (2011).

44. A. Needles et al., "Development and initial application of a fully integrated photoacoustic micro-ultrasound system," IEEE Trans. Ultrason. Ferroelectr. Freq. Control 60(5), 888-897 (2013).

45. T.-H. Bok, E. Hysi, and M. C. Kolios, "Preliminary photoacoustic imaging of the human radial artery for simultaneous assessment of red blood cell aggregation and oxygen saturation in vivo," in IEEE Int. Ultrason. Symp., IEEE, Washington, DC (2017). 
Bok, Hysi, and Kolios: In vivo photoacoustic assessment of the oxygen saturation changes...

46. X. Wang et al., "Noninvasive imaging of hemoglobin concentration and oxygenation in the rat brain using high-resolution photoacoustic tomography," J. Biomed. Opt. 11(2), 024015 (2006).

47. R. Pinto et al., "Feasibility of photoacoustic imaging for the non-invasive quality management of stored blood bags," Vox Sang. 114, 701-710 (2019).

48. M. Lakshman and A. Needles, "Screening and quantification of the tumor microenvironment with micro-ultrasound and photoacoustic imaging," Nat. Methods 12(4), iii-v (2015).

49. H. F. Zhang et al., "Imaging of hemoglobin oxygen saturation variations in single vessels in vivo using photoacoustic microscopy," Appl. Phys. Lett. 90(5), 53901-53903 (2007).

50. Z. Deng and C. Li, "Noninvasively measuring oxygen saturation of human finger-joint vessels by multi-transducer functional photoacoustic tomography," J. Biomed. Opt. 21(6), 061009 (2016).

51. M. Woodward et al., "Associations of blood rheology and interleukin-6 with cardiovascular risk factors and prevalent cardiovascular disease," Br. J. Haematol. 104(2), 246-257 (1999).

52. R. M. Christy et al., "Erythrocyte aggregation and neutrophil function in an aging population," Gerontology 56(2), 175-180 (2010).

53. M. J. Simmonds, H. J. Meiselman, and O. K. Baskurt, "Blood rheology and aging," J. Geriatr. Cardiol. 10(3), 291-301 (2013).

54. R. K. Saha and M. C. Kolios, "A simulation study on photoacoustic signals from red blood cells," J. Acoust. Soc. Am. 129(5), 2935-2943 (2011).

55. E. Hysi, R. K. Saha, and M. C. Kolios, "Photoacoustic ultrasound spectroscopy for assessing red blood cell aggregation and oxygenation," J. Biomed. Opt. 17(12), 125006 (2012).

56. S. Chien et al., "Blood viscosity: influence of erythrocyte deformation," Science 157(3790), 827-829 (1967).

57. N. Tateishi et al., " $\mathrm{O}_{2}$ release from erythrocytes flowing in a narrow $\mathrm{O}_{2}$-permeable tube: effects of erythrocyte aggregation," Am. J. Physiol.—Hear. Circ. Physiol. 281(1), H448H456 (2001).

58. N. Tateishi et al., "Reduced oxygen release from erythrocytes by the acceleration-induced flow shift, observed in an oxygen-permeable narrow tube," J. Biomech. 35(9), 1241-1251 (2002).

59. N. Maeda, "Erythrocyte rheology and oxygen transfer in microcirculatory system," J. Biorheol. 29(1), 2-5 (2015).

60. S. Prahl, "Tabulated molar extinction coefficient for hemoglobin in water," 1999, https:// omlc.org/spectra/hemoglobin/summary.html (accessed 1 June 2020).

61. I. Cerveri et al., "Reference values of arterial oxygen tension in the middle-aged and elderly," Am. J. Respir. Crit. Care Med. 152(3), 934-941 (1995).

62. M. L. Vold et al., "Low oxygen saturation and mortality in an adult cohort: the Troms $\varnothing$ study," BMC Pulm. Med. 15(1), 9 (2015).

63. A. Rodríguez-Molinero et al., "Normal respiratory rate and peripheral blood oxygen saturation in the elderly population," J. Am. Geriatr. Soc. 61(12), 2238-2240 (2013).

64. R. Perez-Padilla et al., "Prevalance of oxygen desaturation and use of oxygen at home in adults at sea level and at moderate altitude," Eur. Respir. J. 27(3), 594-599 (2006).

65. N. Colodny, "Effects of age, gender, disease, and multisystem involvement on oxygen saturation levels in dysphagic persons," Dysphagia 16(1), 48-57 (2001).

66. M. N. Fadhel et al., "Fluence-matching technique using photoacoustic radiofrequency spectra for improving estimates of oxygen saturation," Photoacoustics 19, 100182 (2020).

67. S. Tzoumas et al., "Eigenspectra optoacoustic tomography achieves quantitative blood oxygenation imaging deep in tissues," Nat. Commun. 7, 12121 (2016).

68. F. T. H. Yu and G. Cloutier, "Experimental ultrasound characterization of red blood cell aggregation using the structure factor size estimator," J. Acoust. Soc. Am. 122(1), 645-656 (2007). 
Taehoon Bok is a postdoctoral fellow at Ryerson University. He received his BS and MS degrees in oceanography and ultrasound from Hanyang University in 2004 and 2006, respectively, and his $\mathrm{PhD}$ in ultrasound from Jeju National University in 2011. His current research interests include photoacoustic imaging, hemodynamics, oxygen delivery, and Monte Carlo simulation. He is a member of SPIE.

Eno Hysi received his PhD from the Department of Physics at Ryerson University in May 2020 and is currently a Banting and KRESCENT Fellow at St. Michael's Hospital, Division of Nephrology in Toronto. His research interests lie in the fields of ultrasound and photoacoustic imaging for biomedical applications include cancer treatment monitoring, the study of blood hemodynamics as well as assessing transplant organ quality.

Michael C. Kolios received his BSc degree in physics (minor in computer science) from the University of Waterloo in 1991 and MSc degree and $\mathrm{PhD}$ degrees in medical biophysics from the University of Toronto in 1994 and 1998, respectively. He started his lab at Ryerson University in 1997 and is currently a professor in the Department of Physics and an associate dean, Research and Graduate Studies in the Faculty of Science. His research interests are in biomedical applications of ultrasound and optics. 